\title{
El rol que tiene la influencia familiar y su nivel académico, en los itinerarios curriculares de estudiantes de último curso
}

\section{The role of family and their academic level in final year students' curricular choice}

\author{
Asunción Martínez Martínez \\ asuncionmm@ugr.es \\ FÉlix Zurita Ortega ${ }^{1}$ \\ felixzo@ugr.es \\ Universidad de Granada, España
}

\begin{abstract}
Resumen:
El itinerario curricular que siguen los alumnos de último curso de Bachillerato y Ciclos Formativos es un tema prioritario en el marco educativo, pues de la elección del mismo dependerá su posterior inclusión en el contexto universitario o laboral; asimismo uno de los factores que inciden de manera concisa es el familiar, por tanto planteamos el siguiente estudio con los objetivos de conocer el itinerario curricular de alumnos de último curso de Bachillerato y Ciclos Formativos de Granada así como describir sus características familiares, económicas y sociodemográficas y evaluar la influencia de estas sobre los itinerarios curriculares. Participaron un total de 1.164 alumnos/as, a los que se les registraron parámetros académicos, familiares y laborales, el estudio de los mismos derivó en mostrar homogeneidad en los núcleos familiares en cuanto a estudios e ingresos económicos, con una ligera disparidad entre el empleo de pa-
\end{abstract}

\begin{abstract}
s:
The curricular choice which students make in their last year at High School and Vocational Training is a priority issue in the educational context, since it is going to determine the students' inclusion in the university or employment contexts. Family backgrounds also play an important role in this respect. Bearing this in mind, this study aims to know the curricular choice of final year students (A Level and Vocational Training) in Granada, for the purpose of describing their family, economic and demographic characteristics, and to evaluate the influence of these aspects on the curricular choice they made. A total of 1,164 students, were analyzed in terms of academic success, family and employability. Results show that families had a similar level in terms of academic background and spending power with a slight imbalance between the employment of mothers and fathers. A level students tend to go to university while this proves to be true only
\end{abstract}

1 Dirección para correspondencia (Correspondence address):

Félix Zurita Ortega. Departamento de Didáctica de la Expresión Musical, Plástica y Corporal, Despacho 122.1, Facultad de Ciencias de la Educación de la Universidad de Granada (Spain). 
El rol que tiene la influencia familiar y su nivel académico, en los itinerarios curriculares de estudiantes de último curso

asunción Martínez Martínez y Félix Zurita Ortega

dres y madres; por otra parte los alumnos de Bachillerato se decantan más por su inclusión posterior en el ámbito universitario, mientras los estudiantes de Ciclos Formativos se posicionan de forma similar por estudiar carrera universitaria y acceder al mercado laboral y quedó constatado que los itinerarios curriculares vienen determinados por factores eminentemente familiares, por tanto indicamos la necesidad de estudiar con mayor profundidad a la población de estos cursos, mediante la implementación de programas enfocados a dar a conocer las diversas opciones y a provocar que la elección sea más personal.

\section{Palabras clave:}

Orientación Educativa; Bachillerato; $\mathrm{Ci}$ clos Formativos; Estudiantes; Familia; Académico; Vocación; Universidad; Mercado Laboral; Educación. with half of Vocational Training studentsthe other half wants to join the workforce. It was demonstrated that curricular choice is determined by family factors. Therefore, more attention needs to be paid to these students through the implementation of programmes aimed at highlighting the different options and at making the choice more personal.

\section{Key words:}

Educational Guidance; High School; Vocational Training; Students; Family; Academic; Vocation; University; Job Market; Education.

\section{Résumé:}

L'itinéraire du programme d'études pour les élèves en dernière année de Baccalauréat et de la Formation Professionnelle est une priorité dans le contexte éducatif, car il dépend du choix de l'inclusion ultérieure dans le contexte universitaire ou de l'emploi; également I'un des facteurs qui affecte de manière concise est le facteur familial, par conséquent, nous proposons l'étude suivante avec les objectifs de connaître l'itinéraire du programme d'études des élèves en dernière année de Baccalauréat et de la Formation Professionnelle de Granada (Espagne), ainsi que décrire leur caractéristiques familiales, économiques et sociodémographiques et évaluer l'influence de ces derniers sur les itinéraires du programme d'études. Un total de 1164 élèves, qui ont été enregistrés avec des paramètres académiques, familiaux et professionnels. L'étude de ces paramètres conduit à démontrer l'homogénéité au sein des familles concernant les études et les revenus, avec une légère disparité entre l'emploi des pères et mères; d'autre part, les élèves du Baccalauréat optent pour son inclusion ultérieure au niveau universitaire, tandis que les étudiants de la Formation Professionnelle sont positionnés de façon similaire pour étudier une carrière universitaire et accéder au marché du travail; et on a constaté que les itinéraires du programme d'études sont déterminés par les facteurs éminemment familiaux, donc, nous proposons d'étudier avec une plus grande profondeur la population de ces cours, au moyen de l'implémentation de programmes destinés à présenter les différentes options et à permettre que le choix soit plus personnel.

\section{Mots-clés:}

Orientation Éducative; Baccalauréat; Formation Professionnelle; Étudiants; Famille; Académique; Vocation; Université; Marché du Travail; Éducation.

Fecha de recepción: 22-10-2013

Fecha de aceptación: 2-3-2014 


\section{Introducción}

En el contexto de la sociedad actual existe cada vez una mayor preocupación hacia los sujetos más jóvenes en cuanto a su futuro se refiere. En las décadas anteriores alcanzar la madurez (18 años) implicaba dos vías claramente diferenciadas, por un lado se encontraban aquellos jóvenes que se encaminaban al desarrollo laboral, mientras que otra parte estaban encauzados a la continuación de su formación, pero esto en la actualidad es una paradoja, propiciado por la cada vez mayor carencia de empleo (Viñuela-Jiménez, Rubiera-Morollón \& Cueto, 2010; Ruesga, Martín \& Pérez, 2010 y Blanco, Mercado \& Prado, 2012), este fenómeno de desempleo propiciado por una crisis económica a nivel mundial afecta sobre todo a España que en la actualidad ocupa uno de los primeros lugares en cuanto a tasas de paro se refiere, y ya hace tres años Ruesga et al., (2010) indicaban que la previsión era la de recuperación a nivel mundial exceptuando a Letonia, Lituania, Irlanda, España, Grecia y Chipre; este dato repercute en un exceso de personas que continúan con su formación académica con la finalidad de compensar la deficiencia en términos de experiencia laboral y que la emplean como estrategia para potenciar su formación profesional y cualificación (Gallardo, 2008; GilCalvo, 2009; García-Moreno \& Martínez, 2012) produciendo en ocasiones abandonos de las titulaciones en que se matriculan y generándose el concepto de alargamiento de la juventud (Nascimento, 2012).

La orientación educativa-laboral, es un elemento fundamental en el contexto de la educación, nace para asesorar sobre los itinerarios curriculares del alumnado de enseñanzas medias, considerándose como una herramienta indispensable para determinar el conocimiento de lo que los alumnos desean y pretenden realizar en su futuro a corto y medio plazo, aunque en ciertas ocasiones la vinculación y coordinación entre los Centros de Secundaria y Bachillerato con los Centros Universitarios no es la acorde (Cepero, 2009; Llosa, Acín, Cragnolino \& Del Carmen, 2012; Domínguez, Álvarez \& López, 2013). Si bien como plantea Montero \& Finez (2012), la orientación debe concebirse como un proceso inmerso e inseparable dentro del sistema educativo, mediante la cohesión entre la etapa educativa y la edad escolar.

En el contexto actual en el que nos encontramos, la orientación ha evolucionado conforme a la dinámica y exigencias del mundo moderno, tratando de responder principalmente a la necesidad de ayudar al 
sujeto a la elección libre de una ocupación o profesión, en función de su propia preferencia y aptitudes, entendiéndola Repetto (1994) "como un proceso totalmente pedagógico eliminando cualquier posibilidad de resquicio de una orientación clínica y extraescolar", sin obviar que desde los primeros estudios esta viene apoyándose en el modelo hereditario familiar que el estudiante asumía como indicaban Santana, Feliciano \& Jiménez (2012) o Martínez-Martínez (2013).

Lo que viene a decirnos, que la Orientación, no es solo una guía conductora sino que sirve para que la persona se conozca así mismo e identifique el mundo que le rodea, actuando en calidad de ciudadano responsable, motivado y productivo no solo en una sociedad laboral sino también personal como Mantero (2010) nos señala.

En este sentido se podía entender que la atribución familiar pasaba a un segundo plano en la decisión del hijo, no obstante, debemos cuestionarnos si esta influencia sigue existiendo en este momento y si continúa repercutiendo en su futuro estudiantil y laboral. Bisquerra (1998) añade la caracterización personal como el potencial de cada sujeto, en un proceso de ayuda continúa para fomentar la capacidad de desarrollo en el sujeto en su área personal, escolar, vocacional y/o profesional, a lo largo de toda su vida, y como dicha personalidad vendrá construida por el seno parental, al igual que señalan Santana et al., (2012), cuando señalaban que en los grupos de discusión de su estudio los adolescentes mostraban estereotipos heredados de los progenitores como por ejemplo: "Si quieres estudiar algo de provecho, debes pasar por la Universidad".

Partimos de que la orientación es una disciplina que debe ser tratada desde la infancia dentro del contexto escolar y familiar. Pero no será hasta etapas postobligatorios (Bachillerato y Ciclos Formativos) donde alcanzará su máximo esplendor, así lo ratifican Santana et al., (2012) y Domínguez et al., (2013).

En la actualidad los trabajos realizados sobre este tema giran en torno a la problemática de la escasez de estudios de implementación de programas o bien a la inclusión de menores en riesgo de exclusión (Cantón, 2007; Santana, Feliciano \& Cruz, 2010 o Cavero, 2010), sin embargo antes de la experimentación es necesario conocer que datos existen, en nuestro caso considerábamos importante verificar la influencia que podría tener el contexto familiar en la elección del itinerario académico, dada la escasez de estudios que aglutinaban de forma conjunta a estudiantes de Bachillerato y Ciclos Formativos, la finalidad era constatar 
si los estudiantes de último curso que se encuentran en disposición de acceder a estudios superiores o al mercado laboral se encontraban inducidos por el ámbito familiar, parental o factores económicos, por tanto en relación a este último aspecto, el presente estudio nos aporta cifras sobre lo que acontece en sujetos de 17 a 30 años en relación a la orientación y decisión académica de futuro inmediato por tanto nos hemos planteado como objetivos:

- Determinar el itinerario curricular de alumnos de último curso de Bachillerato y Ciclos Formativos de Grado Superior de Granada así como describir las características familiares, económicas y sociodemográficos.

- Evaluar la influencia familiar, económica y de género en los itinerarios curriculares.

\section{Material y Métodos}

\section{Participantes}

La selección de los participantes se genera del total de 6.132 alumnos/ as matriculados en último curso de Bachillerato y Ciclos Formativos de Granada (España) según datos facilitados por la Consejería de Educación de la Junta de Andalucía durante el curso académico 2012/2013 en la Capital de Granada; para establecer una muestra representativa (error al 0,02; I.C=95,5\%) se emplearon técnicas de estratificación, proporcionalidad y aleatorización, considerándose tipo de rama académica (ciclos formativos y bachillerato) y sexo (hombres-mujeres). Participaron en esta investigación un total de 1.164 estudiantes de Granada (España), con una edad comprendida entre los 17 y 30 años $(M=18,24$ años; DT=1,258), y que presentaron errores muestrales del 0,02 en el total de la muestra y por estratos del 0,03 para Bachillerato y 0,04 para Ciclos Formativos, analizando un total del $18,98 \%$ del universo total. El estudio cumplió con las normas éticas del Comité de Investigación y Declaración de Helsinki de 1975. Se contó en todos los casos con el consentimiento informado de los participantes y se respetó el resguardo a la confidencialidad. 


\section{Diseño}

En este estudio se utilizó un diseño de carácter cuantitativo descriptivo y de tipo transversal. Posteriormente se desarrolló un estudio relacional para analizar el grado de dependencia entre las distintas variables objeto de estudio.

\section{Variables e Instrumentos}

La presente investigación tomó como referencia las siguientes variables e instrumentos de medida, es decir:

- Rama de Estudio, categorizado en Bachillerato y Ciclos Formativos.

- Género, según fuese masculino o femenino.

- Características Familiares, siguiendo las aportaciones de Cepero (2009) y Martínez-Martínez (2013) obtenidas a través de preguntas de autoregistro todas ellas fiables y validadas por los autores anteriormente mencionados sobre:

- Nivel Académico de Padres/Madres que nos establecían cinco niveles académicos: No presencia, Estudios de Primaria, Secundaria, Formación Profesional y Universitarios.

- Ocupación Laboral de Padres y Madres, categorizado en cinco niveles: Trabajadores no Cualificados, Trabajadores Cualificados en una Profesión hasta Oficiales de Primera, Dueños de Empresas Familiares de cinco o menos empleados y Trabajadores Autónomos, Empresarios con empleados de cinco a diez y Diplomados Universitarios y por ultimo Empresarios con más de diez empleados y Titulados Universitarios Superiores.

- Ingresos Económicos mensuales en el domicilio familiar, distribuidos en siete categorías: menos de 500€, entre 500€-1000€, entre $1000 €-1500 €$, de $1500 €-2000 €$, entre $2000 €-2500 €$, de $2500 €-3000 €$ y más de $3000 €$ al mes.

- Itinerarios Curriculares, obtenido a través de preguntas de autoregistro, estructurado en ocho categorías siguiendo las aportaciones de los Planes de Estudios del MEC: B-U-ML, B-CF-ML, B-ML, BP-U-ML, CF-U-ML, CF-B-ML, CF-B-U-ML, CF-ML. Donde "B" es Bachillerato, " $\mathrm{U}$ " es Universidad, "CF" es Ciclo Formativo y "ML" es Mercado Laboral. 


\section{Procedimiento}

En primer lugar, a través de la Facultad de Ciencias de la Educación de la Universidad de Granada (Área de Corporal), y en contacto con la Delegación de Educación de la Junta de Andalucía, se solicitó la colaboración de los Centros Educativos de la Capital de Granada seleccionados. La dirección de cada Centro Educativo fue informada sobre la naturaleza de la investigación y solicitando la colaboración de sus alumnos/as. En segundo lugar se adjunto un modelo de autorización destinado a los responsables legales de los adolescentes pidiéndoles su consentimiento informado.

En todo momento se garantizó a los participantes el anonimato de la información recogida aclarando que su utilización sería sólo con fines científicos. Los encuestadores estuvieron presentes durante la recogida de los datos para poder resolver cualquier duda al respecto. Esta se desarrolló sin ningún tipo de problema o anormalidad a reseñar. En último lugar se agradeció a los docentes, orientadores y responsables su colaboración y se les informó del envío en un futuro próximo de un informe sobre los datos obtenidos respetando la confidencialidad de los mismos.

\section{Análisis de los Datos}

Para el análisis de los datos se utilizó el programa estadístico SPSS 20.0., mediante la utilización de descriptivos, medias y ANOVA para establecer las diferencias entre las distintas variables: Género, Rama Académica, Familiares e Itinerarios Curriculares.

\section{Resultados}

Tomando como referencia los análisis descriptivos, concernientes a los 1.164 participantes, respecto a las variables seleccionadas, se constató que $488(41,9 \%)$ jóvenes eran varones y $676(58,1 \%)$ eran féminas. La mayor parte de ellos se encontraban estudiando Bachillerato (58,6\%; $\mathrm{n}=682)$, y los restantes $482(41,4 \%)$ cursaban Ciclos Formativos de Grado Superior, asimismo en lo que respecta a los parámetros de tipo familiar como queda reflejado en la tabla 1, tanto en el caso de los padres como de las madres poseían estudios de Secundaria y Universitarios, en 
El rol que tiene la influencia familiar y su nivel académico, en los itinerarios curriculares de estudiantes de último curso

asunción Martínez Martínez y Félix Zurita Ortega

cuanto a la ocupación de las madres (40\%) eran trabajadoras no cualificadas mientras que los padres en un 39,9\% ocupaban puestos cualificados en una profesión. Por último en lo que respecta a los ingresos económicos percibidos el 68,4\% se situaban entre los 500 y 2000 euros mensuales.

Tabla 1. Descriptivos de los parámetros objeto de estudio.

\begin{tabular}{|c|c|c|}
\hline \multicolumn{3}{|c|}{ Descriptivos } \\
\hline \multirow[t]{2}{*}{ Género } & Masculino & $41,9 \%(n=488)$ \\
\hline & Femenino & $58,1 \%(n=676)$ \\
\hline \multirow{2}{*}{$\begin{array}{l}\text { Rama de } \\
\text { Estudio }\end{array}$} & Bachillerato & $58,6 \%(n=682)$ \\
\hline & Ciclos Formativos & $41,4 \%(n=482)$ \\
\hline \multirow{2}{*}{$\begin{array}{c}\text { Ingresos } \\
\text { Económicos }\end{array}$} & Menos 500 Euros & $4,8 \%(n=56)$ \\
\hline & Entre 501 Euros-1000 Euros & $19,8 \%(n=231)$ \\
\hline & Entre 1001 Euros-1500 Euros & $26,6 \%(n=310)$ \\
\hline & Entre 1501 Euros- 2000 Euros & $21,8 \%(n=254)$ \\
\hline & Entre 2001 Euros- 2500 Euros & $14,3 \%(n=166)$ \\
\hline & Entre 2501 Euros-3000 Euros & $6,4 \%(n=75)$ \\
\hline & Mayor 3000 Euros & $6,2 \%(n=72)$ \\
\hline \multirow{6}{*}{$\begin{array}{c}\text { Nivel } \\
\text { Académico }\end{array}$} & & Padres Madres \\
\hline & No Sabe/No Contesta & $\begin{array}{l}1,5 \% \\
(n=17)\end{array}$ \\
\hline & Primaria & $\begin{array}{cc}18,6 \% & 17,5 \% \\
(n=217) & (n=204)\end{array}$ \\
\hline & Secundaria & $\begin{array}{cc}30,8 \% & 32,3 \% \\
(n=358) & (n=376)\end{array}$ \\
\hline & Formación Profesional & $\begin{array}{cc}21,9 \% & 19,7 \% \\
(n=255) & (n=229)\end{array}$ \\
\hline & Universidad & $\begin{array}{cc}27,2 \% & 28,9 \% \\
(n=317) & (n=336)\end{array}$ \\
\hline
\end{tabular}


El rol que tiene la influencia familiar y su nivel académico, en los itinerarios curriculares de estudiantes de último curso Asunción Martínez Martínez y Félix Zurita Ortega

\begin{tabular}{|c|c|c|c|}
\hline \multirow[t]{6}{*}{$\begin{array}{l}\text { Ocupación } \\
\text { Laboral }\end{array}$} & No Padre/Madre & $\begin{array}{l}1,5 \% \\
(n=17)\end{array}$ & $\begin{array}{l}1,6 \% \\
(n=19)\end{array}$ \\
\hline & Trabajadores No Cualificados & $\begin{array}{l}15,7 \% \\
(n=183)\end{array}$ & $\begin{array}{l}40,0 \% \\
(n=466)\end{array}$ \\
\hline & Trabajadores Cualificados en una Profesión & $\begin{array}{l}39,9 \% \\
(n=465)\end{array}$ & $\begin{array}{c}28,0 \% \\
(n=326)\end{array}$ \\
\hline & Trabajadores Autónomos o Pequeño Comercio & $\begin{array}{l}23,0 \% \\
(n=268)\end{array}$ & $\begin{array}{l}12,6 \% \\
(n=147)\end{array}$ \\
\hline & $\begin{array}{c}\text { Comerciantes, Pequeños Empresarios y } \\
\text { Diplomados }\end{array}$ & $\begin{array}{l}7,2 \% \\
(n=84)\end{array}$ & $\begin{array}{c}8,8 \% \\
(n=103)\end{array}$ \\
\hline & $\begin{array}{l}\text { Empresarios, Directivos y Titulados Universi- } \\
\text { tarios Superiores }\end{array}$ & $\begin{array}{l}12,6 \% \\
(n=147)\end{array}$ & $\begin{array}{c}8,8 \% \\
(n=103)\end{array}$ \\
\hline
\end{tabular}

En lo que respecta a los itinerarios curriculares se constató que los itinerarios más valorados por los alumnos en un 45,5\% $(n=530)$ fue el de B-U-ML, seguido de CF-U-ML (20,9\%; $n=243)$ y CF-ML (19,4\%; $n=226)$, mientras los otros tres apenas fueron puntuados (Tabla 2)

Tabla 2. Itinerarios curriculares del alumnado de último curso

Itinerario Curricular

Frecuencia Porcentaje

\begin{tabular}{|c|c|c|}
\hline Bachillerato/Universidad/Mercado Laboral (B-U-ML) & 530 & $45,5 \%$ \\
\hline Bachillerato/Ciclo Formativo/Mercado Laboral(B-CF-ML) & 66 & $5,7 \%$ \\
\hline $\begin{array}{l}\text { Bachillerato/ Ciclo Formativo /Universidad/Mercado } \\
\text { Laboral (B-CF-U-ML) }\end{array}$ & 69 & $5,9 \%$ \\
\hline Bachillerato/Mercado Laboral(B-ML) & 17 & $1,5 \%$ \\
\hline Ciclo Formativo /Universidad/Mercado Laboral(CF-U-ML) & 243 & $20,9 \%$ \\
\hline Ciclo Formativo /Bachillerato/Mercado Laboral(CF-B-ML) & 5 &, $4 \%$ \\
\hline $\begin{array}{l}\text { Ciclo Formativo /Bachillerato/Universidad/Mercado } \\
\text { Laboral(CF-B-U-ML) }\end{array}$ & 8 &, $7 \%$ \\
\hline Ciclo Formativo /Mercado Laboral(CF-ML) & 226 & $19,4 \%$ \\
\hline Total & 1164 & $100,0 \%$ \\
\hline
\end{tabular}

Al establecer las relaciones entre las variables de tipo género y rama de estudios con los itinerarios curriculares no se establecieron diferencias estadísticamente significativas entre la elección de la vía curricular y el género $(p=.113)$, si se encontraron en relación a la procedencia de estudios $(p=.000)$, de esta manera los estudiantes de Ciclos Formativos 
en un 46,9\% preferían la opción de Mercado Laboral frente al 2,5\% de alumnos/as de Bachillerato, asimismo en la opción de acceso directo a la universidad y posteriormente al mercado laboral, el $77,7 \%$ de los alumnos/as de Bachillerato contemplaban esta opción como prioritaria frente al 50,4\% de los Ciclos Formativos. (Tabla 3).

Tabla 3. Género y rama según elección del Itinerario curricular de último curso

\begin{tabular}{cccc}
\hline & \multicolumn{2}{c}{ Modalidades } & \multirow{2}{*}{$\mathbf{X}^{2}$} \\
\hline Género & Masculino & Femenino & \\
\hline B-U-ML & $43,2 \%(n=211)$ & $47,2 \%(n=319)$ & $\mathrm{p}=.113$ \\
B-CF-ML & $5,7 \%(\mathrm{n}=28)$ & $5,6 \%(\mathrm{n}=38)$ & \\
B-CF-U-ML & $4,3 \%(\mathrm{n}=21)$ & $7,1 \%(\mathrm{n}=48)$ & \\
B-ML & $1,8 \%(\mathrm{n}=9)$ & $1,2 \%(\mathrm{n}=8)$ & \\
CF-U-ML & $21,9 \%(\mathrm{n}=107)$ & $20,1 \%(\mathrm{n}=136)$ & \\
CF-B-ML &, $2 \%(\mathrm{n}=1)$ &, $6 \%(\mathrm{n}=4)$ & \\
CF-B-U-ML &, $4 \%(\mathrm{n}=2)$ &, $9 \%(\mathrm{n}=6)$ & \\
CF-ML & $22,3 \%(\mathrm{n}=109)$ & $17,3 \%(\mathrm{n}=117)$ & \\
Rama & Ciclo Formativo & Bachillerato & $\mathbf{X}^{2}$ \\
\hline U-ML & $50,4 \%(\mathrm{n}=243)$ & $77,7 \%(\mathrm{n}=530)$ & $\mathrm{p}=.000$ \\
B/CF-ML & $1,0 \%(\mathrm{n}=5)$ & $9,7 \%(\mathrm{n}=66)$ & \\
B/CF-U-ML & $1,7 \%(\mathrm{n}=8)$ & $10,1 \%(\mathrm{n}=69)$ & \\
ML & $46,9 \%(\mathrm{n}=226)$ & $2,5 \%(\mathrm{n}=17)$ & \\
\hline
\end{tabular}

En el estudio en cuanto a los itinerarios se detectan diferencias $(p=.000)$ en lo concerniente al nivel académico del padre y madre, la ocupación laboral de ambos e ingresos económicos; teniendo en cuenta los tres itinerarios más valorados por los participantes (B-U-ML; CF-UML y CF-ML), las discrepancias en el primer caso vienen establecidas porque en los estudiantes de B-U-ML, el nivel académico de los padres y madres en la mayoría de los casos es de procedencia universitaria $(56,5 \%$ y $58 \%$ ) frente a un escaso $32 \%$ de estudios de Primaria; en el segundo itinerario (CF-U-ML), la mayor procedencia es de nivel de Primaria (cifras en torno al 29\% en ambos) y en CF-ML, también tienen estudios de nivel de Primaria.

Respecto a la ocupación laboral los datos arrojaron que en el caso de participantes de B-U-ML, sus padres tenían en mayor ocupación la de Empresarios, Directivos y Titulados Universitarios Superiores en cifras 
superiores al $60 \%$, en el caso de CF-U, la mayoría (cifras superiores al $20 \%$ ) procedían de Trabajadores no Cualificados al igual que sucedía en CF-ML. Por último los ingresos económicos arrojaron valores muy dispares, así los ingresos de los alumnos/as de B-U-ML se situaban entre los 1000 y 2500 euros en proporciones similares (en torno al 20\%), mientras que CF-U-ML oscilaba entre 500-1500 euros (valores del 30\%) y en CFML la franja de 1000 a 1500 euros en un $31 \%$. Tabla 4.

Tabla 4. Factores familiares según elección del Itinerario curricular

\begin{tabular}{|c|c|c|c|c|}
\hline Modalidad & & B-U-ML & CF-U-ML & CF-ML \\
\hline \multicolumn{5}{|c|}{ NIVEL ACADEMICO } \\
\hline \multirow{2}{*}{ No Sabe/No Contesta } & Padre & $29,4 \%(n=5)$ & $17,6 \%(n=3)$ & $35,3 \%(n=6)$ \\
\hline & Madre & $15,8 \%(n=3)$ & $36,8 \%(n=7)$ & $31,6 \%(n=6)$ \\
\hline \multirow{2}{*}{ Primaria } & Padre & $32,7 \%(n=71)$ & $28,6 \%(n=62)$ & $27,6 \%(n=60)$ \\
\hline & Madre & $32,8 \%(n=67)$ & $29,9 \%(n=61)$ & $28,4 \%(n=58)$ \\
\hline \multirow{2}{*}{ Secundaria } & Padre & $41,3 \%(n=148)$ & $24,3 \%(n=87)$ & $20,9 \%(n=75)$ \\
\hline & Madre & $39,9 \%(n=150)$ & $23,1 \%(n=87)$ & $22,3 \%(n=84)$ \\
\hline \multirow{2}{*}{ Formación Profesional } & Padre & $49,8 \%(n=127)$ & $17,6 \%(n=45)$ & $18,0 \%(n=46)$ \\
\hline & Madre & $50,2 \%(n=115)$ & $15,7 \%(n=36)$ & $17 \%(n=39)$ \\
\hline \multirow{2}{*}{ Universidad } & Padre & $56,5 \%(n=179)$ & $14,5 \%(n=46)$ & $12,3 \%(n=39)$ \\
\hline & Madre & $58 \%(n=195)$ & $15,5 \%(n=52)$ & $11,6 \%(n=39)$ \\
\hline \multicolumn{5}{|c|}{ OCUPACION LABORAL } \\
\hline \multirow{2}{*}{ No Padre/Madre } & Padre & $29,4 \%(n=5)$ & $17,6 \%(\mathrm{n}=3)$ & $35,3 \%(n=6)$ \\
\hline & Madre & $15,8 \%(n=3)$ & $36,8 \%(n=7)$ & $31,6 \%(n=6)$ \\
\hline \multirow{2}{*}{$\begin{array}{l}\text { Trabajadores No Cuali- } \\
\text { ficados }\end{array}$} & Padre & $31,7 \%(n=58)$ & $28,4 \%(n=52)$ & $26,2 \%(n=48)$ \\
\hline & Madre & $37,3 \%(n=174)$ & $26,6 \%(n=124)$ & $21,9 \%(n=102)$ \\
\hline \multirow{2}{*}{$\begin{array}{l}\text { Trabajadores Cualifica- } \\
\text { dos en una Profesión }\end{array}$} & Padre & $43,9 \%(n=204)$ & $22,8 \%(n=106)$ & $17,8 \%(n=83)$ \\
\hline & Madre & $47,2 \%(n=154)$ & $18,4 \%(n=60)$ & $20,2 \%(n=66)$ \\
\hline \multirow{2}{*}{$\begin{array}{l}\text { Trabajadores Autóno- } \\
\text { mos o Pequeño Comer- } \\
\text { cio }\end{array}$} & Padre & $46,6 \%(n=125)$ & $19 \%(n=51)$ & $23,5 \%(n=63)$ \\
\hline & Madre & $49 \%(n=72)$ & $17 \%(n=25)$ & $15,6 \%(n=23)$ \\
\hline \multirow{2}{*}{$\begin{array}{c}\text { Comerciantes, Pe- } \\
\text { queños Empresarios y } \\
\text { Diplomados }\end{array}$} & Padre & $57,1 \%(n=48)$ & $8,3 \%(n=7)$ & $14,3 \%(n=12)$ \\
\hline & Madre & $58,3 \%(n=60)$ & $14,6 \%(n=15)$ & $16,5 \%(n=17)$ \\
\hline \multirow{2}{*}{$\begin{array}{c}\text { Empresarios, Directivos } \\
\text { y Titulados Universita- } \\
\text { rios Superiores }\end{array}$} & Padre & $61,2 \%(n=90)$ & $16,3 \%(n=24)$ & $9,5 \%(n=14)$ \\
\hline & Madre & $65 \%(n=67)$ & $11,7 \%(n=12)$ & $11,7 \%(n=12)$ \\
\hline
\end{tabular}




\begin{tabular}{cccc}
\hline \multicolumn{4}{c}{ INGRESOS ECONOMICOS } \\
\hline Menos 500 Euros & $3 \%(n=16)$ & $5,8 \%(n=14)$ & $8 \%(n=18)$ \\
Entre 501 Euros-1000 Euros & $13 \%(n=69)$ & $31,3 \%(n=76)$ & $22,1 \%(n=50)$ \\
Entre 1001 Euros-1500 Euros & $22,8 \%(n=121)$ & $30 \%(n=73)$ & $30,1 \%(n=68)$ \\
Entre 1501 Euros- 2000 Euros & $23,2 \%(n=123)$ & $19,3 \%(n=47)$ & $20,4 \%(n=46)$ \\
Entre 2001 Euros- 2500 Euros & $20,8 \%(n=110)$ & $6,6 \%(n=16)$ & $9,7 \%(n=22)$ \\
Entre 2501 Euros-3000 Euros & $8,7 \%(n=46)$ & $3,3 \%(n=8)$ & $6,2 \%(n=14)$ \\
Mayor 3000 Euros & $8,5 \%(n=45)$ & $3,7 \%(n=9)$ & $3,5 \%(n=8)$ \\
\hline
\end{tabular}

\section{Discusión}

En este estudio de 1164 participantes de último curso de Bachillerato y Ciclos Formativos de Grado Superior, es de las mismas características que otros trabajos realizados en la geografía española (Rodríguez-Moreno, 1986; Naranjo, 1993; Toscano, 2004; Cepero, 2009; Sanjuán, 2010), e incluso se señala la importancia de estudios de esta índole (Castilla, 2003; Capilla, 2009) y que estos se realicen desde el contexto escolar (la escuela).

Debemos puntualizar que una parte muy numerosa de la muestra recogida provenían de Bachillerato mientras que de los Ciclos Formativos nos encontramos con un alto absentismo de contestación hacía las preguntas realizadas, esto concuerda con lo reportado por Toscano (2004) que matiza que entre los estudiantes de Bachillerato todo lo relacionado con conocer el futuro de demandas universitarias, tutoriales y de organización les crea numerosas atenciones, sin embargo en el contexto de los Ciclos Formativos estas expectativas no son tan altas por lo que en ocasiones deciden no acudir a encuestas de esta índole.

En cuanto a los resultados de tipo familiar, la mayoría de padres y madres poseían el nivel académico de secundaria, seguido de la formación universitaria, asimismo se determinó que los padres formaban una unidad familiar homogénea en cuanto al nivel académico de los mismos. El entorno de socialización y aprendizaje es mostrado por los progenitores ya que son los que transmiten valores, hábitos, estilos de vida y comportamientos, constituyendo los principales modelos de conducta hasta la adolescencia (Burunat, 2004; Carrasco, 2004; Sadurní \& Rostan, 2004). Alonso \& Ramón (2005) muestran la importancia del estilo educativo 
familiar, ya que enseñan con su ejemplo y los hijos/as aprenden por imitación (Proyecto Hombre, 2000). Martín del Buey, Fernández, Martín, Dapelo, Marcote \& Granados (2008) exponen que los padres son los primeros maestros en el reto de la educación de sus hijos.

Al igual que señalan Cortés \& Conchado (2012) se hace implícito el paternalismo, donde los varones ocupan puestos más relevantes y con mejores sueldos. En este estudio apreciamos como los padres se posicionan como Trabajadores Cualificados en una Profesión y Trabajadores Autónomos o del Pequeño Comercio, mientras que las madres se señalaban como Trabajadoras no Cualificadas destacando que aquí se englobaban a las amas de casa. Al hilo de esto Gil (2004), en su trabajo de mujeres adultas de Sevilla, afirma que la mano de obra femenina se concentra en el empleo sumergido y en concreto en el sector servicios donde desempeñan ocupaciones no cualificadas y Winkler, Magaña \& Wolf (2001) y Cepero (2009), argumentan que las personas logran las diversas posiciones de prestigio por variadas razones, muchas de las cuales tienen que ver con factores políticos, tradiciones profesionales y valores sociales, que son los condicionantes que provocan el mérito o excelencia en el trabajo. Respecto a los ingresos económicos que se reciben en el domicilio familiar siete de cada diez señalaban percibir entre 500 Euros y 2000 Euros.

La ocupación laboral del padre y de la madre de los participantes granadinos se encuentra asociada al nivel académico alcanzado, así los trabajadores no cualificados poseían en la mayoría de los casos estudios de nivel primario, mientras que en cuanto a la distribución de comerciantes, pequeños empresarios o diplomados y directivos o titulados superiores presentan estudios de carácter universitario lo que confirma que a mayor nivel académico mayor es la ocupación laboral, al igual que señalan Ruiz-Carrascosa \& Molero (2010), y que los ingresos económicos también van en concordancia con las ocupaciones laborales por lo que a mayor nivel de ocupación los ingresos económicos son mayores, datos opuestos a lo que se observa en trabajadores no cualificados o trabajadores cualificados en una sola profesión con los salarios más bajos, sin embargo en la actualidad son los estamentos universitarios los que muestran una mayor preocupación ante la situación de que un titulado universitario no alcance la demanda laboral que se le presupone. (Cajide, Porto, Abeal, Peláez \& Zamora, 2000; Ayats, 2001; Rodríguez-Espinar, 2003; Aguado, 2006; Luque, Del Barrio, Sánchez \& Ibáñez, 2008; Moyano, 2008; Ruiz-Carrascosa \& Molero, 2010). 
Los alumnos de Bachillerato en el itinerario curricular se decantan más por su inclusión posterior en el ámbito universitario, dándonos estos datos la confirmación de que estos estudios están encaminados a que el alumno/a continúe su formación de índole universitaria; asimismo los estudiantes de Ciclos Formativos también pretenden estudiar carrera universitaria, por lo que suponemos que se matriculan en los Grados Superiores para tener después acceso a la formación de tipo universitaria para poder escoger las plazas que se ofertan en los diversos grados. Otra de las opciones contempladas es la del acceso directo al mercado laboral, itinerario básico de los Ciclos Formativos.

Cepero (2009) señala que los sujetos en Ciclos Formativos en muchos casos tienen claro la opción de seguir estudiando debido a que parte de este alumnado realiza los módulos con la intención de alcanzar la media para poder entrar a estudios universitarios, bien porque no la consiguió por la vía de Bachillerato, COU o Selectividad o por no enfrentarse a este camino que considera más duro y largo, con el incentivo de tomar un itinerario académico más fácil, y esto le aporta además un título que le cualifica para incorporase al mundo laboral directamente, en caso de no poder entrar a la diplomatura que desean. Al hilo de todo esto Casal, García \& Merino (2005), señalan cuatro itinerarios formativos, entre los que indica itinerarios de ausencia de titulación básica que jóvenes sin Educación Secundaria Obligatoria se presentan al mercado de trabajo como candidatos a ocupar trabajos no cualificados y expuestos a la vulnerabilidad, los itinerarios de pobreza formativa, jóvenes que van al mercado de trabajo con el graduado escolar o la formación profesional de primer nivel (con o sin acreditación profesional); los itinerarios propios de las enseñanzas medias de bachillerato o de formación profesional superior y que incluye universitarios sin acreditación y por último los itinerarios universitarios, aunque con suerte muy desigual tanto en las edades de titulación como en la transición al trabajo.

No se determinaron diferencias por género pero si por rama donde se destaca que la mayoría de los alumnos/as de Bachillerato pretendían seguir con estudios universitarios, mientras que la mitad de los estudiantes de Ciclos Formativos lo que perseguían era el acceso al mercado laboral de forma directa desde la modalidad de la que se procede, y la otra mitad buscaba la opción de acceso directo a la universidad y posteriormente al mercado laboral, este hecho corrobora que el alumno/a de Bachillerato busca esencialmente prepararse para integrarse en la co- 
munidad educativa, como señalan entre otros Corominas \& Isus (1998) y Toscano (2004), y que explican el proceso trascendental que supone esta elección.

Mientras que en el caso de los Ciclos Formativos se ha cambiado la tendencia, pues antiguamente su funcionalidad era la de preparar a las personas para desempeñar un oficio o tarea laboral, y en la actualidad se ha cambiado la dinámica y numerosos estudiantes matriculados lo hacen con la finalidad de acceder a la Universidad, bien porque no tienen nota y desde el grado superior se ofertan plazas para acceder a la Universidad constituyendo otra opción, o bien porque buscan la posibilidad de tener otros estudios (Bachillerato, Grado Superior y Titulación Universitaria), pues como dicen numerosos autores estamos en una etapa donde los jóvenes deben formarse pues las expectativas de empleo no existen e incluso la formación es esencial pues en el caso de encontrar empleo este ya debe estar respaldado de una titulación ya que como plantean González-Pienda, Núñez, González-Pumariega \& Álvarez (2001) y Martín del Buey et al., (2008) el tipo de motivación que predomina en el sujeto viene determinada entre otros factores por el autoconcepto, metas de aprendizaje y las expectativas de logro. De todo esto detectamos que entre el alumnado de ciclos formativos que pretenden estudiar su itinerario formativo está claro y bien encauzado, sus preferencias se dirigen hacia un futuro universitario y una seguridad en su elección postsecundaria, como se señaló anteriormente.

En nuestro trabajo quedó constatado que los itinerarios curriculares vienen determinados por factores eminentemente familiares, de esta manera el alumnado de Bachillerato que pretende continuar sus estudios en la Universidad presenta un perfil familiar de padres/madres con titulación Universitaria y que ocupan laboralmente puestos de directivos, empresarios o de titulación superior con el consiguiente incremento en los ingresos económicos respecto a los otros itinerarios, aspecto inversos a lo que se apreció en los otras vías estudiadas. Esto no hace más que confirmar lo reportado por Cohler \& Altergott (1995); Lopata (1995); Phillipson (1997) o Duque (2002) que en sus teorías orientadas a los roles familiares dejan claro que estos poseen diferentes roles, que concuerdan con diversos sectores, y esto explica la direcciones a tomar, así citaban que de padres titulados universitarios los hijos iban encaminados al Bachiller y después a la Universidad, mientras que de familias de trabajadores cualificados o no cualificados (electricistas, mecánicos, 
albañiles,...) su destino eran los Ciclos Formativos (antigua Formación Profesional), para aprender y dedicarse al igual que sus padres al oficio que este desempeñaba.

Para finalizar debemos señalar que como en todo estudio se presentan una serie de limitaciones, en este han venido producidas porque una parte considerable de los estudiantes de Ciclos Formativos rehusaron la realización de este estudio, del mismo modo también podríamos haber incluido cuestiones hacia los padres para constatar si la información recogida era la veraz y tener en cuenta algunos supuestos como abandono de titulación universitaria en los últimos cursos y motivos de la misma.

\section{Conclusiones}

Como principales conclusiones de este estudio extraemos que:

- La mayoría de padres y madres poseían el nivel académico mínimo de Secundaria y formaban una unidad familiar homogénea en cuanto al nivel académico y económico, asimismo los padres se posicionan como Trabajadores Cualificados en una Profesión y Trabajadores Autónomos o del Pequeño Comercio, mientras que las madres se señalaban como Trabajadoras no Cualificadas destacando que aquí se englobaban a las amas de casa, asociada al nivel académico alcanzado.

- Los alumnos de Bachillerato se decantan más por su inclusión posterior en el ámbito universitario, mientras los estudiantes de Ciclos Formativos se posicionan de forma similar por estudiar carrera universitaria y acceder al mercado laboral

- En nuestro trabajo quedó constatado que los itinerarios curriculares vienen determinados por factores eminentemente familiares.

\section{Referencias Bibliográficas}

Aguado, P.M. (2006). Empleo universitario en Aragón 2005. Zaragoza: UNIVERSA.

Alonso, J. \& Ramón, J. (2005). Prácticas educativas familiares y autoestima. Psicothema, $17(1), 76-82$.

Ayats, J.C. (2001). El empleo de los titulados de la Universidad Politécnica de Valencia. Valencia: Universidad Politécnica de Valencia. 
El rol que tiene la influencia familiar y su nivel académico, en los itinerarios curriculares de estudiantes de último curso Asunción Martínez Martínez y Félix Zurita Ortega

Bisquerra, R. (1998). Modelos de orientación e intervención psicopedagógica. Barcelona: Praxis.

Blanco, A., Mercado, C. \& Prado, A. (2012). Perfil y motivación de la juventud española emprendedora. Revista de Estudios sobre Juventud, 99,22-34.

Burunat, E. (2004). El desarrollo del sustrato neurobiológico de la motivación y emoción en la adolescencia: ¿un nuevo periodo crítico?. Infancia y aprendizaje, 27(1), 87-104.

Cajide, J., Porto, A., Abeal, C., Peláez, M. \& Zamora, E. (2000). La calidad universitaria y transición al empleo de los graduados, en Cajide, J., Santos, M.A. \& Porto, A. (coords), Calidad educativa y empleo en contextos multiculturales. Santiago de Compostela: ICE de la Universidad de Santiago de Compostela.

Cantón, I. (2007). La escuela orientadora. La acción tutorial desde una perspectiva institucional. Educatio Siglo XXI, 25, 237-240.

Capilla, R. (2009). Análisis estratégico de los estudios TIC en la Universidad Politécnica. Tesis Doctoral: Universidad Politécnica de Valencia.

Carrasco, A. (2004). Consumos de alcohol y estilos de vida: una tipología de los adolescentes españoles. Revista de Psicología Social, 19(1), 51-79.

Casal, J., García, M. \& Merino, R. (2007). Los sistemas educativos comprensivos ante las vías y los itinerarios formativos. Revista de Educación, 342, 213-237.

Castilla, M.T. (2003). Perfil profesional e itinerarios de formación e inserción profesional del psicopedagogo. Tesis Doctoral: Universidad de Granada.

Cavero, M. Á. B. (2010). Estudio investigación valorativa de la eficacia del Programa de Refuerzo, Orientación y Apoyo (PROA) en alumnos de Educación Secundaria Obligatoria. Revista de Educación, 352, 405-429.

Cepero, B.A. (2009). Las preferencias profesionales y vocacionales del alumnado de secundaria y formación profesional específica. Tesis Doctoral: Universidad de Granada.

Cohler, B. \& Altergott, K. (1995). The family of second holf life: connecting theories and finding. En R. Blüssun \& U. H. Bedford (Ed.), Handbook of aging and the family. Werport, C.T.: Greenwood Press.

Corominas, A. \& Isus, S. (1998). Transiciones y orientación. Revista de Investigación Educativa, 16(2), 155-184.

Cortés, P.A. \& Conchado, A. (2012). Los contextos parentales y académicos y los valores laborales en la toma de decisiones en bachillerato. ESE. Estudios sobre Educación, 22, 93-114.

Domínguez, G., Álvarez, F.J. \& López, A.M. (2013). Acción tutorial y orientación en el periodo de transición de la Educación Secundaria a la Universidad. La orientación en el alumno de nuevo ingreso. REDU, Revista de Docencia Universitaria, 11(2), 221-241.

Duque, P. (2002). Representaciones sociales de roles en la vejez: una comparación transcultural. Revista Latinoamericana de Psicología, 1-2(34), 95-106.

Gallardo, J. (2008). Juventud, trabajo, desempleo e identidad: un enfoque psicosocial. Tesis doctoral: Universidad Complutense de Madrid.

García-Moreno, J.M. \& Martínez, R. (2012). Ser joven hoy en España. Dificultades para el acceso al mercado de los adultos. BARATARIA: Revista castellano-manchega de Ciencias Sociales, 14, 29-40. 
El rol que tiene la influencia familiar y su nivel académico, en los itinerarios curriculares de estudiantes de último curso

asunción Martínez Martínez y Félix Zurita Ortega

Gil, R. (2004). Detención de las necesidades de asesoramiento como medida para la inclusión ocupacional femenina. Conferencia Internacional Orientación, Inclusión Social y Desarrollo de la Carrera. La Coruña.

Gil-Calvo, E. (2009). Trayectorias y transiciones. ¿Qué rumbos?. Revista de Estudios de Juventud, 87(9), 15-29.

González-Pienda, J., Núñez, J., González-Pumariega, S. \& Álvarez, L. (2001). Estrategias de aprendizaje, género y rendimiento académico. Revista Gallego-Portuguesa de Psicología y Educación, 5(7), 41-58.

Llosa, S., Acín, A., Cragnolino, E., \& Del Carmen, M. (2012). La demanda potencial y la demanda efectiva en educación de jóvenes y adultos: primera aproximación al enfoque cuantitativo y cualitativo de la biografía educativa. Reflexiones en torno al significado atribuido a la educación y la capacitación laboral. Cuadernos de Educación, (2).

Lopata, H. Z. (1995). Feminist perspectives on social gerontology. En R. Bliesznes, y V. H. Bedford (Eds.), Handbook of aging and the family. Westport, CT: Greenwood Press.

Luque, T., Del Barrio, S., Sánchez, J. \& Ibáñez, J.A. (2008). Estudio de egresados de la Universidad de Granada. Años 2004-2005. Granada: Universidad de Granada.

Mantero, M. (2010). Orientación educativa y culturas docentes en la educación postobligatoria: estudio de las relaciones entre el orientador y el profesorado en niveles de formación profesional específica. Tesis Doctoral: Universidad de Huelva.

Martín del Buey, F., Fernández, A., Martín, E., Dapelo, B., Marcote, R. \& Granados, P. (2008). Cuestionario de personalidad eficaz para la formación profesional. Psicothema, 20(2), 224-228.

Martínez-Martínez, A. (2013). La orientación como actividad educativa y vocacional en los itinerarios curriculares del alumnado de bachiller y formación Profesional y su inclusión en el Mercado Laboral. Tesis doctoral: Universidad de Granada.

Montero, A.J. \& Silva, M.J. (2012). Competencias básicas: Un marco legislativo, teorico y aplicativo. International Journal of Development and Educational Psychology: INFAD, $1(2), 1-10$.

Moyano, J. (2008). Estudio de la inserción laboral de los titulados de la Universidad de Jaén. Informe 2008. Jaén: Universidad de Jaén.

Naranjo, M.A. (1993). Estilo cognitivo, intereses y elección académico profesional en la orientación de los alumnos de COU. Tesis Doctoral: Universidad Complutense de Madrid.

Nascimento, R. (2012). El alargamiento de la juventud: un análisis psicosocial de las trayectorias de jóvenes de Brasil y España. Tesis Doctoral: universidad complutense de Madrid.

Phillipson, C. (1997). Social relationship in later life: a review of the research literature. International Journal of Geriatric Psychiatry, 12, 505-512.

Proyecto Hombre (2000). "Entre todos" .Programa de prevención escolar y familiar de drogodependencias. Madrid: Asociación Proyecto Hombre.

Repetto, E. (1994). Orientación educativa e intervención psicopedagógica. Madrid: UNED.

Rodríguez-Espinar, S. (2003). Educacio superior i treball a Catalunya: estudi de la inserció laboral dels graduats de las universitats publiques catalanes. Barcelona: AQSUC. 
El rol que tiene la influencia familiar y su nivel académico, en los itinerarios curriculares de estudiantes de último curso asunción Martínez Martínez y Félix Zurita Ortega

Rodríguez-Moreno, M.L. (1986). Orientación profesional y acción tutorial en las enseñanzas medias. Madrid: Narcea.

Ruesga, S., Martín, J.L. \& Pérez, L. (2010). Informe de coyuntura sobre el mercado de trabajo en España y Andalucía (Primer trimestre de 2010). Revista de Temas Laborales, 105, 131-159.

Ruiz-Carrascosa, J. \& Molero, D. (2010). Ocupación laboral y formación complementaria de los titulados universitarios. Bordón, 62(1), 109-122.

Sadurní, M. \& Rostan, C. (2004). La importancia de las emociones en los periodos sensibles del desarrollo. Infancia y aprendizaje, 27 (1), 105-114.

Sanjuán, M.M. (2010). Los contenidos y las competencias del módulo de formación y orientación laboral (FOL): visión del profesorado de Galicia. Revista de Innovación Educativa, 20, 89-103.

Santana, L.E., Feliciano, L.A. \& Cruz, A.E. (2010). El Programa de Orientación Educativa y Sociolaboral: un instrumento para facilitar la toma de decisiones en Educación Secundaria. Revista de Educación, 351, 73-105.

Santana, L.E., Feliciano, L.A. \& Jiménez, A.B. (2012). Toma de decisiones y género en Bachillerato. Revista de Educación, 359, 357-387.

Toscano, J.M. (2004). Estudio sobre la orientación académica para el tránsito del Bachillerato a la Universidad de Huelva: diseño, aplicación y evaluación de un programa de orientación para el acceso a la Universidad. Tesis Doctoral: Universidad de Huelva.

Viñuela-Jiménez, A., Rubiera-Morollón, F. \& Cueto, B. (2010). An analysis of urban size and territorial location effects on employment probabilities: the Spanish case. Growth and Change. A Journal of Urban and Regional Policy, 41(4), 495-519.

Winkler, M., Magaña I. \& Wolf, X. (2001). Mujeres en la historia de la Psicología: Autorías y Paradojas. Revista Latinoamericana de Psicología, 1(33), 23-37. 
